\title{
Self-assembly of self-propelled magnetic grains
}

\author{
Marcos A. Madrid ${ }^{1,2, *}$, Ramiro M. Irastorza ${ }^{1,2, * *}$, Ariel G. Meyra ${ }^{1,2, * * *}$, and C. Manuel Carlevaro ${ }^{1,2, * * * *}$ \\ ${ }^{1}$ Instituto de Física de Líquidos y Sistemas Biológicos CONICET - CCT La Plata, La Plata, Argentina. \\ ${ }^{2}$ Departamento de Ingeniería Mecánica, UTN - FRLP, Berisso, Argentina.
}

\begin{abstract}
In this work, we study bidisperse mixtures of self-propelled magnetic particles of different shapes via discrete element method simulations. We show how these particles self-assemble into clusters and how these clusters depend on the ratio of the mixture, the magnetic interaction, and the shape of the grains. It is found that the mix ratio of the system controls the cluster size. Besides, the intensity of the magnetic dipoles and the shape of the grains in the mixture rule the average number of neighbors in contact and the shape of the clusters. By varying the intensity of the interactions, globular, linear and branched clusters were obtained.
\end{abstract}

\section{Introduction}

The advances made in the last decade in the field of active matter gave rise to very important applications in selfpropelled systems [1-4]. How these systems self-organize or self-assemble is a topic of great interest [5, 6]. Applications of these ideas have been extended to active granular matter $[7,8]$. These materials can self-assemble in different patterns or clusters of different shapes and sizes [9, 10].

Another aspect of interest is to understand how granular matter self-organizes when in addition to the contact forces, a longer range force is added, for example, magnetic interaction $[11,12]$.

In this work, we present 2D discrete element simulations of magnetic disks and triangles. We perform simulations of binary mixtures of self-propelled grains with different permanent magnetic dipoles (repulsive/attractive). We analyze the final configuration after a long period of time of each simulated system using tools from network theory when varying particle types, mix ratio and interaction intensities.

\section{Model and simulated systems}

\subsection{The model}

The system consists of a 2D forty-sided regular polygon platform of area $A$ ( $x y$ plane) over which we randomly place binary mixtures of 300 perfectly rigid grains. The grains are disks or regular (equilateral) triangles of the same area $a$ in order to keep the packing fraction constant and we vary the proportion of each type of grain in the binary mixture. We also define two different magnetic dipoles, vertically oriented and antiparallel (positive

\footnotetext{
*e-mail: mmadrid@iflysib.unlp.edu.ar

**e-mail: rirastorza@iflysib.unlp.edu.ar

***e-mail: agmeyra@iflysib.unlp.edu.ar

****e-mail: manuel@iflysib.unlp.edu.ar

A video is available at https://doi.org/10.48448/bprj-9z04
}

and negative direction of $z$-axis, respectively) which produces an attractive force between the particles of different types, and a repulsive force when the particles are of the same type. By varying the ratio between them, we vary the forces intensity.

\subsection{Interaction forces and system dimensions}

The non-contact interaction force between two grains due to magnetic dipoles is given by:

$$
\vec{F}_{m_{\alpha \beta}}=\frac{3 \mu_{0} \overrightarrow{m_{\alpha}} \vec{m}_{\beta}}{4 \pi\left|r_{\alpha \beta}\right|^{5}} \overrightarrow{r_{\alpha \beta}},
$$

with $\alpha, \beta=1,2$, and $\overrightarrow{m_{\alpha}}=\mu_{\alpha} \overrightarrow{\mu_{\alpha}}, \overrightarrow{m_{\beta}}=\mu_{\beta} \overrightarrow{\mu_{\beta}}$, being $\overrightarrow{\mu_{\alpha}}, \overrightarrow{\mu_{\beta}}$ a unitary vector and $\mu_{\alpha}$ and $\mu_{\beta}$ the intensity of their magnetic dipole, $\mu_{0}=4 \pi \times 10^{-7} \mathrm{H} \cdot \mathrm{m}^{-1}$ and $\overrightarrow{r_{\alpha \beta}}$ is the vector that connects the center of the two given magnetic particles. Additionally, the friction forces grain-grain, grainwall, and grain-base are also considered.

The reference system is composed of binary mixtures of $N=300$ magnetic disks, with $N_{1}$ disks that have $\vec{m}_{1}$ and $N_{2}$ have $\overrightarrow{m_{2}}$ magnetic dipole, being $\overrightarrow{m_{1}}$ and $\overrightarrow{m_{2}}$ antiparallel. The diameter of the disks are $d$, and initially they are randomly placed in the simulation box. The forty-sided polygon is circumscribed in a circle of diameter $D=45 \mathrm{~d}$. We consider the weight of each disk as a reference force $F_{w}=m g$, where $m$ is the mass and $g$ the gravity constant. Consequently, the friction force, the force due to the impulses, and the magnetic force are defined as: $F_{f}=v F_{w}$, $F_{i m p}=5.41 F_{w}$, and $F_{m_{\alpha \beta}}=1.62 F_{w} \mu_{\alpha} \mu_{\beta} r_{\alpha \beta}^{\vec{*}} /\left|r_{\alpha \beta}^{\vec{*}}\right|^{5}$ (where $\left.r_{\alpha \beta}^{\vec{*}}=\overrightarrow{r_{\alpha \beta}} / d\right)$, respectively. It is remarked that triangles and disks have the same friction force with the base because they have the same area and density.

The simulation time step is set to $d t=$ $0.003132 \sqrt{(d / g)} \mathrm{s}$ and the simulations were stopped when the total kinetic energy was less than $0.135 F_{w} d$ Joule or simulation time extends to $10^{6} d t$. The friction 
coefficient between grains, and between grains and the base and walls is set to $v=0.5$. The coefficient of restitution is 0.1 .

For each simulation run, the particles of each mixture, disks-disks (DD), triangles-triangles (TT), or their mixtures (DT and TD), are randomly located in the simulation box. Each particle is self-propelled as it is periodically subjected to impulses in a given fixed direction. After a given time-lapse, $31.32 \sqrt{(d / g)}$, these impulses are stopped and the system is allowed to relax. The data is then extracted from that final relaxed configuration. As we have already mentioned, we carried out all the simulations keeping the packing fraction constant $\phi=N a / A=0.149$ and we change the mix ratio of particles $\phi_{1} / \phi_{2}$, being $\phi_{i}=N_{i} a / A, i=1,2$.

We used Box2D for the DEM simulations [13], which has been successfully used to study granular flow [14], clogging [15], force networks [16], tapping [17], granular dampers [18], stick-slip [19], and soil mechanics [20]. This software library uses a constraint solver to handle contact and collision dynamics of rigid bodies. For each time step, an iterative algorithm is used to solve constraints on overlaps and friction between bodies through a Lagrange multiplier scheme [20,21]. The Coulomb criterion is implemented for the solid friction for grain-grain, grainwall and grain base interactions, with the static and dynamic coefficients having equal numerical values. Energy dissipation is considered using a restitution coefficient in

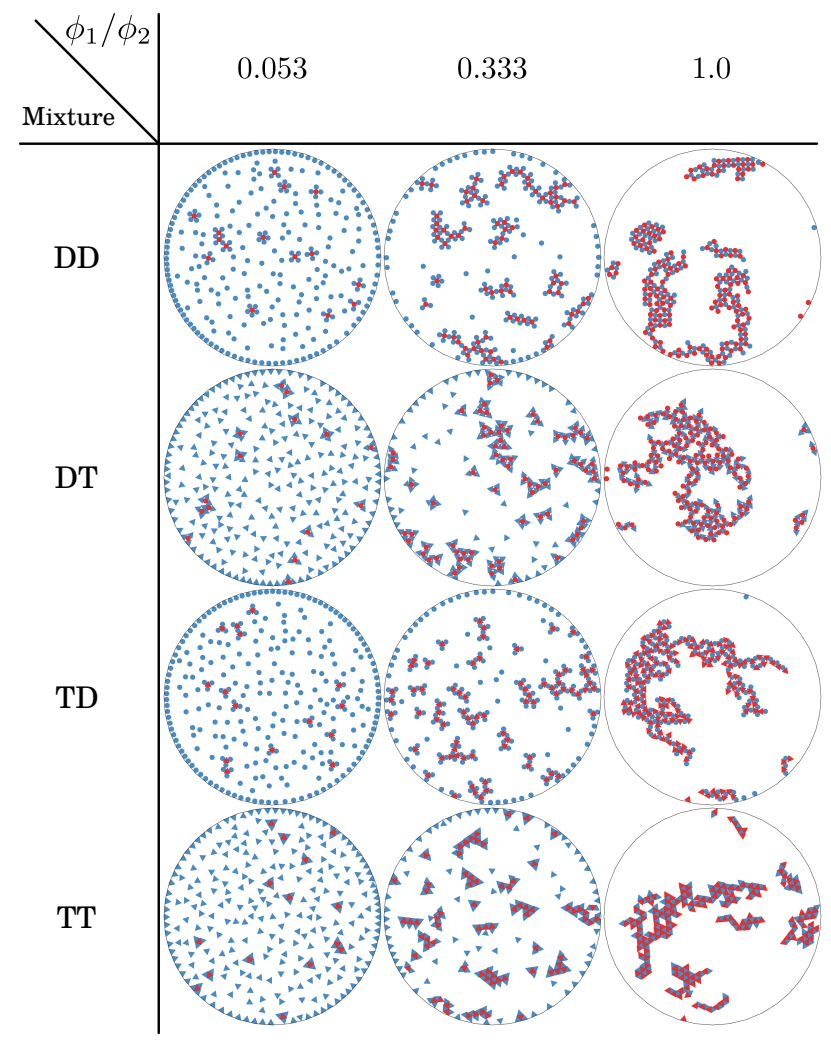

Figure 1: Snapshots for $\mu_{1} / \mu_{2}=1$, and three mix ratios of each simulated mixtures.

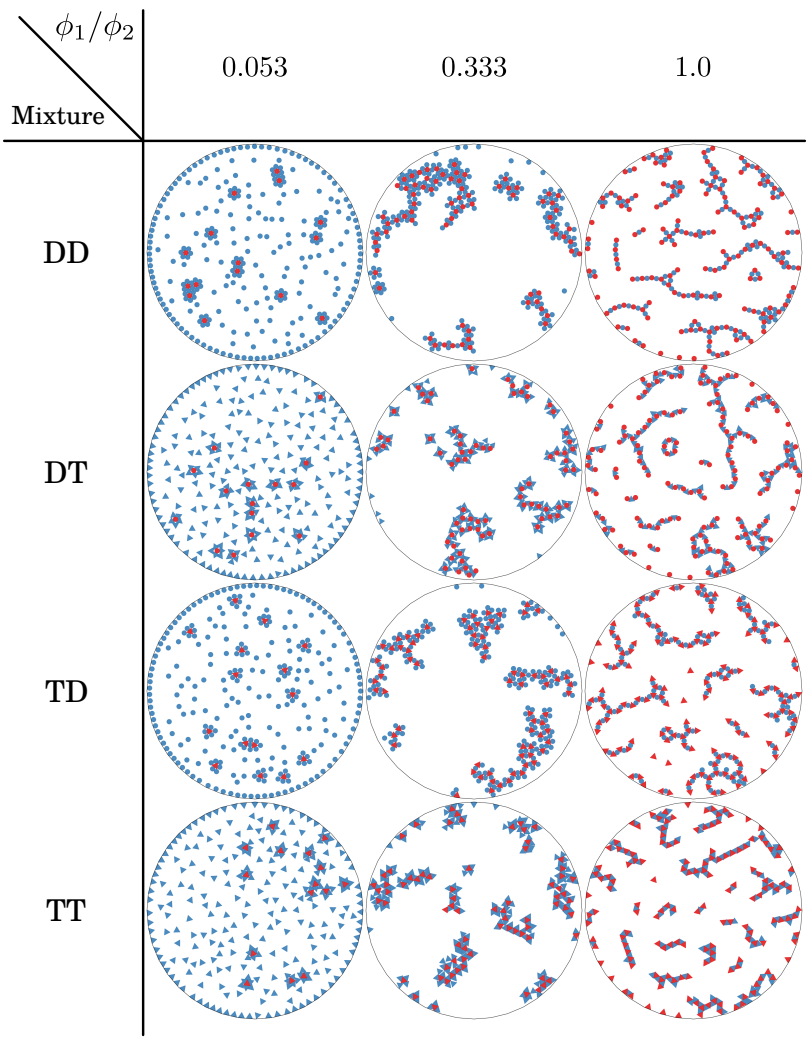

Figure 2: Snapshots for $\mu_{1} / \mu_{2}=5$, and three mix ratios of each simulated mixtures.

the collision solver. After computing all the contact forces, the Newton-Euler equations of motion are integrated, forward in time, using a symplectic Euler algorithm [21].

\section{Results and discussion}

Self-assembled clusters are analyzed using graphs theory. For each cluster, we built a graph where the nodes are the grains (of type 1 or 2), and an edge will be created for each pair of grains in contact. The degree of a particular node $i$ can be computed as:

$$
k_{i}=\sum_{j=1}^{N} A_{i j}
$$

being $A_{i j}$ the elements of the adjacency matrix $\mathbf{A}$. The element $A_{i j}$ is 1 if the particles $i$ and $j$ are in contact and 0 otherwise. $N$ is the total number of nodes. An important property is the global mean degree of the network:

$$
\langle k\rangle=\sum_{i} k_{i}
$$

Thereby, $\langle k\rangle$ is also known as the average osculation or kissing number [22]. The maximum value for a monodisperse mixture of disks in 2D systems is $\langle k\rangle=6$. Summarizing, $\left\langle k_{i}\right\rangle$ accounts for the mean number of grains that are in touch with grains of type $i$. 
In this section, we study the effect on the final selfassembled structures due to: shapes of the grains, magnetic interactions ratio, and mix ratio. Analyzed shapes are disks, triangles, and their mixtures, intensities of interaction are $\mu_{2}=1$ and $1 \leq \mu_{1} / \mu_{2} \leq 5$, and mix ratio are $0.053 \leq \phi_{1} / \phi_{2} \leq 1.0$ (see Figs. 1 and 2 ). For each setup configuration, we ran a set of 50 simulations with different initial seeds. The results presented here are the average over these sets of simulations.
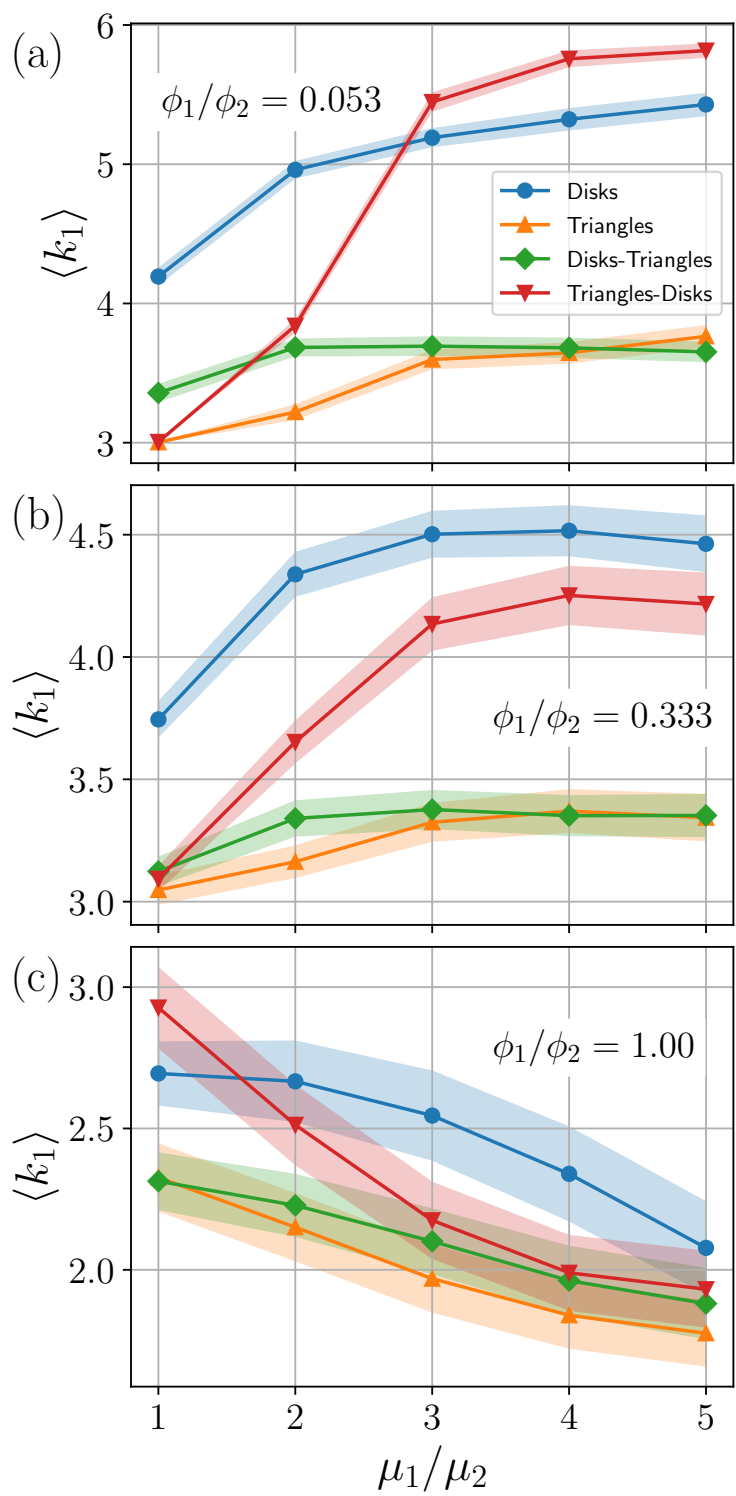

Figure 3: Mean degree for particles of type 1 as a function of $\mu_{1} / \mu_{2}$, for the three mix ratios values $\phi_{1} / \phi_{2}$ simulated: a) 0.053 , b) 0.333 and c) 1.00 . Shaded areas denote standard deviation of the mean.

It is worth remembering that magnetic interactions are repulsive for grains of the same type, while grains of different type are attracted each other $\left(F_{m_{11}}\right.$ and $F_{m_{22}}$ are repulsive, but $F_{m_{12}}$ and $F_{m_{21}}$ are attractive).

As a reference system, we now discuss a mixture of $N_{1}$ disks with $\overrightarrow{\mu_{1}}=(0,0,1)$ and $N_{2}$ disks with $\overrightarrow{\mu_{2}}=(0,0,-1)$. In Fig. 1, there are snapshots for three different $\phi_{1} / \phi_{2}$ when $\mu_{1} / \mu_{2}=1.0$ while in Fig. 2 can be seen snapshots for the same three mix ratios but $\mu_{1} / \mu_{2}=5.0$.

For low values of $\phi_{1} / \phi_{2}$ (for all simulated $\mu_{1} / \mu_{2}$ ratios), the self-assembled clusters are mainly monomers or dimmers, i. e.: clusters composed by one grain of type 1 surrounded by grains of type 2 (see blue curve in Fig. 3a). However, it can be seen that the mean degree $\left\langle k_{1}\right\rangle$ increases as the ratio $\mu_{1} / \mu_{2}$ does. This tendency can be observed for the value $\phi_{1} / \phi_{2}=0.333$, nevertheless, this radically changes when $\phi_{1} / \phi_{2} \rightarrow 1.0$, in fact it is reversed, because clusters become larger but linear (compare first row (DD), third column $\left(\phi_{1} / \phi_{2}=1\right)$ panel of Fig.1 with the same of Fig.2). This behavior is a consequence of the two competing effects: the repulsion between grains of the same type and the attraction when they are of a different type. Besides, if the stoichiometric relationship $\left(\phi_{1} / \phi_{2}\right)$ is the appropriate, the assembled cluster can achieve the maximum degree (in the case of DD mixtures is $k_{1}=6$ ). This is only possible if the attraction force $F_{m_{12}}$ overcomes the repulsion force $F_{m_{22}}$ ). Conversely, if the repulsion $F_{m_{11}}$ is weak, as it is when $\mu_{1} / \mu_{2}=1.0$, the clusters are globular (see Fig.1 panel DD row for $\left.\phi_{1} / \phi_{2}=1\right)$. When $\mu_{1} / \mu_{2}=1.0$ and $\phi_{1} / \phi_{2} \rightarrow 1.0$ clusters are globular with a square arrangement, but for $\mu_{1} / \mu_{2} \rightarrow 5.0$ clusters become linear (see Fig. 2 panel DD row for $\phi_{1} / \phi_{2}=1$ ). Then, keeping $F_{m_{22}}$ constant, when $\mu_{1} / \mu_{2} \rightarrow 5.0$, the value of $F_{m_{11}}$ increases and this determines the shape of the clusters. All the simulated mixtures show nearly the same behavior, but with subtle differences between them. In the case of TT and DT mixtures, $\left\langle k_{1}\right\rangle$ is never greater than 4 , being the DT mixtures the cases which show minor variation in $\left\langle k_{1}\right\rangle$. For the TD mixtures, systems with small $\phi_{1} / \phi_{2}$ and $\mu_{1} / \mu_{2}=1.0$, the value of $\left\langle k_{1}\right\rangle$ is nearly 3 , however it sharply increases when $\mu_{1} / \mu_{2}>3.0$, reaching their maximum values. This behaviour is due to a synergetic effect between geometry, interactions and packing fraction. The attractive magnetic force is maximum when a given disk is in contact with a triangle in the middle of its side. It is energetically favoured to set three disks in each side of a given triangle (maximizing the attraction). Then, other

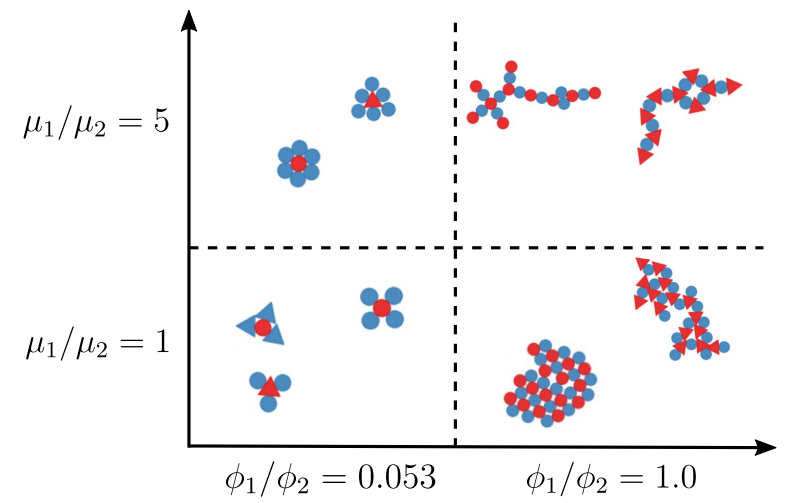

Figure 4: Some representative self-assembly structures obtained in the simulated systems, which depend on the value of the control parameters: grain shape, mix ratios and intensities of the magnetic force. 
three disks can be easily located at the vertices of the triangle due to the generated well in the potential energy landscape produced by the magnetic interaction. It must be noticed that, disks attached to the triangle are not in contact with each other. This situation minimizes the repulsion potential energy. The excess of disks, type 2, around each triangle $\left(\phi_{1} / \phi_{2} \ll 1.0\right)$ completes the necessary conditions to produce a mixture with a larger kissing number.

\section{Conclusions}

In this work, we study three important factors that govern the self-assembly of self-propelled magnetic grains: mix ratio, dipolar interaction, and shape.

Regarding mix ratio, for small ratios of $\phi_{1} / \phi_{2}$, it is more likely to find monomers and dimers than any other structures. Bigger clusters are obtained if this ratio is increased. The dipolar interactions could drive the kissing number $\left\langle k_{1}\right\rangle$. This can be observed, for instance, when $\mu_{1} / \mu_{2} \rightarrow 1.0$ the values of $\left\langle k_{1}\right\rangle$ are next to 3 for all the simulated mixtures. However, when $\mu_{1} / \mu_{2} \rightarrow 5.0$ the value of $\left\langle k_{1}\right\rangle$ rises up to 6 , which is the maximum kissing number in 2D for monodisperse disks systems, while in the case of triangle mixtures the maximum number of neighbours in contact can be 12 (for TT), but it is not energetically favorable. Regarding the shape, it allows controlling the variation of $\left\langle k_{1}\right\rangle$ in the case of pure disks or triangles-disks mixtures, while for pure triangles and disks-triangles cases $\left\langle k_{1}\right\rangle$ is lower than 4.0 and remains roughly constant, independently of $\phi_{1} / \phi_{2}$ and $\mu_{1} / \mu_{2}$.

The implication of the present research is that the assembled structures (see Fig. 4) can be tuned by choosing the correct mix ratio and interaction. The shape (disks or triangles) can be used to modify the probability of finding a particular pattern and/or a local arrangement (i. e.: square or triangular clusters). Future research should consider the potential effects of different shapes, and study a wider set of parameters.

\section{Acknowledgements}

This work has been partially supported by CAFCI (Argentine-France), UTN (Argentine) through Grant PID MAUTNLP0006542 and CONICET (Argentine) through Grant PUE 201822920180100010 CO. AGM y CMC has received funding from the European Union's Horizon 2020 research and innovation programme under the Marie Skłodowska-Curie grant agreement No. 734276 (CONIN).

\section{References}

[1] A. Pena-Francesch, J. Giltinan, M. Sitti, Nat. Commun. 10, 3188 (2019)

[2] B. Kherzi, M. Pumera, Nanoscale 8, 17415 (2016)

[3] J. Katuri, X. Ma, M.M. Stanton, S. Sánchez, Accounts Chem. Res. 50, 2 (2017)

[4] R. Zimroz, J. Wodecki, R. Król, M. Andrzejewski, P. Sliwinski, P. Stefaniak, Self-propelled mining machine monitoring system - data validation, processing and analysis, in Mine Planning and Equipment Selection (Springer, 2014), p. 1285

[5] M. Gusenbauer, H. Nguyen, F. Reichel, L. Exl, S. Bance, J. Fischbacher, H. Özelt, A. Kovacs, M. Brandl, T. Schrefl, Physica B 435, 21 (2014)

[6] R. Amirifar, K. Dong, Q. Zeng, X. An, Soft Matter 15, 5933 (2019)

[7] L. Walsh, C.G. Wagner, S. Schlossberg, C. Olson, A. Baskaran, N. Menon, Soft Matter 13, 8964 (2017)

[8] C. Scholz, S. Jahanshahi, A. Ldov, H. Löwen, Nat. Commun. 9, 5156 (2018)

[9] R. Sánchez, P. Díaz-Leyva, Physica A 499, 11 (2018)

[10] S. Miyashita, Z. Nagy, B. Nelson, R. Pfeifer, Entropy 11, 643 (2009)

[11] L. Abelmann, T.A.G. Hageman, P.A. Löthman, M. Mastrangeli, M.C. Elwenspoek, Science Advances 6, 1 (2020)

[12] U. Culha, Z.S. Davidson, M. Mastrangeli, M. Sitti, PNAS 117, 11306 (2020)

[13] Box2d physics engine, https://www.box2d.org, accessed: 2020-10-16

[14] E. Goldberg, C.M. Carlevaro, L.A. Pugnaloni, Pap. Phys. 7, 070016 (2015)

[15] E. Goldberg, C.M. Carlevaro, L.A. Pugnaloni, J. Stat. Mech.: Theory Exp. 2018, 113201 (2018)

[16] L.A. Pugnaloni, C.M. Carlevaro, M. Kramár, K. Mischaikow, L. Kondic, Phys. Rev. E 93, 062902 (2016)

[17] R.M. Irastorza, C.M. Carlevaro, L.A. Pugnaloni, J. Stat. Mech.: Theory Exp. 2013, P12012 (2013)

[18] M. Sánchez, C.M. Carlevaro, L.A. Pugnaloni, J. Vib. Control 20, 1846 (2013)

[19] C.M. Carlevaro, R. Kozlowski, L.A. Pugnaloni, H. Zheng, J.E.S. Socolar, L. Kondic, Phys. Rev. E 101, 012909 (2020)

[20] M. Pytlos, M. Gilbert, C.C. Smith, Géotech. Lett. 5, 243 (2015)

[21] J. Bender, K. Erleben, J. Trinkle, Comput. Graph. Forum 33, 246 (2014)

[22] J.H. Conway, Sphere packings, lattices, codes, and greed, in Proceedings of the International Congress of Mathematicians (Springer, 1995), p. 45 